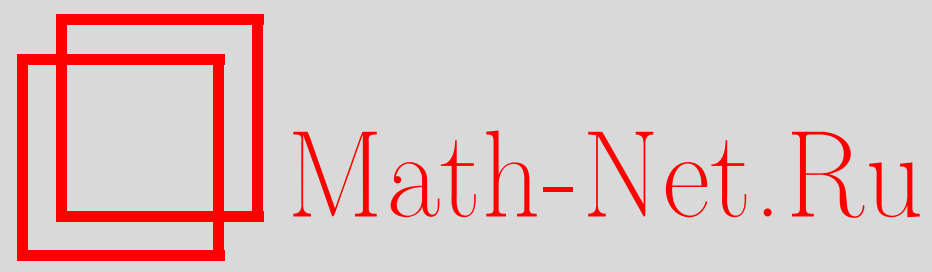

А. А. Приходько, Стохастические конструкции перемешивающих систем положительного локального ранга, Матем. заметки, 2001, том 69, выпуск 2, 316-319

DOI: https://doi.org/10.4213/mzm684

Использование Общероссийского математического портала Math-Net.Ru подразумевает, что вы прочитали и согласны с пользовательским соглашением http://www . mathnet.ru/rus/agreement

Параметры загрузки:

IP : 3.80 .253 .173

26 апреля 2023 г., 06:31:16

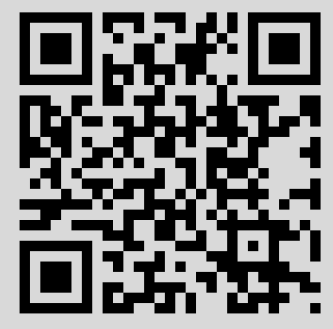




\section{СТОХАСТИЧЕСКИЕ КОНСТРУКЦИИ ПЕРЕМЕШИВАЮЩИХ СИСТЕМ ПОЛОЖИТЕЛЬНОГО ЛОКАЛЬНОГО РАНГА}

\section{А. А. Приходько}

Автоморфизм $T$ пространства Лебега $(X, \mathscr{A}, \mu), \mu(X)=1$, имеет ранг 1 , если существует последовательность разбиений $\xi_{n}=\left\{C_{n}, T C_{n}, T^{2} C_{n}, \ldots, T^{h-1} C_{n}, Y_{n}\right\}$, сходящаяся к разбиению на точки (это означает, что любое измеримое множество сколь угодно точно приближается $\xi_{n}$-измеримыми множествами). Автоморфизм назьвается перемешивающим, если для любых измеримых множеств $A$ и $B$ выполнено $\mu\left(T^{t} A \cap B\right) \rightarrow \mu(A) \mu(B)$ при $n \rightarrow \infty$. Пример перемешивающего автоморфизма $T$ ранга 1 приведен в работе Орнстейна [1]. Им было доказано, что $T$ не имеет собственных факторов и коммутирует только со своими степенями. Позднее методами теории джойнингов были обнаружены другие интересные свойства преобразований такого типа.

Идея Орнстейна состоит в "рандомизации" общей конструкции преобразования ранга 1 (см., например, [2]). Тогда если параметры конструкции распределены достаточно случайно, то полученная система является перемешивающей почти наверное. Известно, что автоморфизмы Орнстейна (почти наверное) обладают сингулярным максимальным спектральным типом (см. работы Абдалави [2] и Бургена [3]).

Мы предлагаем новую стохастическую конструкцию перемешивающих систем (автоморфизмов и потоков) ранга 1 , а также систем положительного локального и бесконечного ранга, обладающих рядом дополнительных метрических свойств. В нашей конструкции достаточные условия перемешивания имеют явное выражение через статистические параметры системы (см. теорему 1). Все результаты работы формулируются для потоков, аналогичные теоремы верны и для автоморфизмов.

Основные резульаты теории систем конечного ранга изложены в обзоре [4].

\section{1. Перемешивающие системы ранга 1.}

ОПРЕдЕЛЕния. Назовем пару $(U, \varphi)$ башней высоты $h$ относительно измеримого потока $\left(T^{t}\right)$ на пространстве Лебега $(X, \mathscr{A}, \mu)$, если $U \in \mathscr{A}$ и отображение $\varphi: U \rightarrow[0, h]$ обладает следующими свойствами:

а) отображение $\varphi$ измеримо: для любого $B \in \mathscr{B}[0, h]$ множество $\varphi^{-1} B \in \mathscr{A}$, где $\mathscr{B}[0, h]-$ $\sigma$-алгебра борелевских подмножеств $[0, h]$;

b) $T^{t} \varphi^{-1} B=\varphi^{-1} S^{t} B$, если $B$ - борелевское подмножество $[0, h]$ и $S^{t} B \subseteq[0, h]$, где $S^{t} x=x+t$.

Скажем, что поток $\left(T^{t}\right)$ имеет $\beta$-ранг, если сушествует последовательность башен $\left(U_{n}, \varphi_{n}\right)$ со следующими свойствами:

a) $\mu\left(U_{n}\right) \rightarrow \beta$ при $n \rightarrow \infty$;

b) для любого измеримого множества $A$ найдется последовательность множеств $\widetilde{A_{n}} \in \mathscr{A}\left(\varphi_{n}\right)$ такая, что $\mu\left(A \triangle \widetilde{A}_{n}\right) \rightarrow 0$, когда $n \rightarrow \infty$, где $\mathscr{A}\left(\varphi_{n}\right)$ есть $\sigma$-алгебра множеств вида $\varphi^{-1}(B) \cup E$, где $B \in \mathscr{B}\left[0, h_{n}\right], E \subseteq X \backslash U_{n}$.

Число $\beta(T)$, равное супремуму всех $\beta$, удовлетворяющих сформулированным условиям, называют локальным рангом потока. Говорят, что $\left(T^{t}\right)$ - поток ранга 1 , если $\left(T^{t}\right)$ имеет $\beta$-ранг с $\beta=1$.

Конструкция потока ранга 1 . На шаге 0 рассмотрим множество вида $U_{0}=C_{0} \times\left[0, h_{0}\right]$, где $C_{0}=\left[0, \alpha_{0}\right]$ и $\alpha_{0}>0$. Зададим наш поток как специальный поток над $C_{0}$. Это означает, что точка $(c, y)$ под действием потока движется равномерно и прямолинейно в вертикальном направлении до тех пор, пока $y$ не окажется равным $\rho_{0}(c)$ (функция $\rho_{0}$ называется функцией возвращения), а затем мгновенно "перескакивает" в точку $S_{0}(c)$, где $S_{0}$ - некоторый автоморфизм пространства

Работа выполнена при поддержке Российского фонда фундаментальных исследований, грант № 99-01-01104, и Гранта государственной поддержки научных школ, грант № 96-15-96135. 
$\left(C_{0}, \lambda\right)$ и $\lambda$ - мера Лебега. Таким образом, фазовое пространство $X=\left\{(c, y): 0 \leqslant y \leqslant \rho_{0}(c)\right.$, $\left.c \in C_{0}\right\}$, а мера $\mu$ с точностью до нормирующего множества совпадает с мерой Лебега. Разделим отрезок $C_{0}$ на $q_{0}$ равных отрезков $C_{0,1}, \ldots, C_{0, q_{0}}$ (мы пренебрегаем концами отрезков, поскольку все объекты рассматриваются с точностью до множеств меры 0 ). Определим функцию возвращения на всех отрезках, кроме последнего, следующим образом: $\rho_{0}(c)=h_{0}+s_{0, j}$, если $c \in C_{0, j}$, где $s_{0, j}$ - заданные положительные числа. При этом потребуем, чтобы $S_{0}(c)$ равнялось $c+\alpha_{0} / q_{0}$ при $c \notin C_{0, q_{0}}$. Заметим, что $S_{0}\left(C_{0, j}\right)=C_{0, j+1}$, если $j<q_{0}$.

На шаге 1 мы доопределим функцию возвращения и автоморфизм $S_{0}$ "почти на всем" отрезке $C_{0, q_{0}}$. Заметим, что поскольку множества $C_{0, j}, j=2, \ldots, q_{0}$, являются итерациями под действием $S_{0}$ множества $C_{0,1}$, задание функции $\rho_{0}$ эквивалентно заданию функции возвращения $\rho_{1}$ относительно отрезка $C_{0,1}$. Точнее, положим $C_{1}=C_{0,1}=\left[0, \alpha_{1}\right]$ и рассмотрим наш поток как специалный над $C_{1}$ с функцией возвращения $\rho_{1}$ и автоморфизмом $S_{1}$. При этом

$$
\rho_{1}(c)=h_{1}+\rho_{0}\left(S^{q_{0}-1} c\right), \quad S_{1}=S_{0}^{q_{0}}, \quad h_{1}=q_{0} h_{0}+\sum_{j} s_{0, j} .
$$

Как и раньше, поделим отрезок $C_{1}$ на $q_{1}$ равных частей и определим $\rho_{1}$ на отрезке $C_{1, j}$ как $h_{1}+s_{1, j}$, а автоморфизм $S_{1}$ пусть, как и раньше, осуществляет сдвиг на $\alpha_{1} / q_{1}$ вправо, если $c \notin C_{1, q_{1}}$. Повторяя эту конструкцию, мы полностью определим как функцию возвращения $\rho_{0}$, так и $S_{0}$. Нетрудно доказать, что построенный поток является потоком ранга 1 с последовательностью аппроксимирующих башен $\left(U_{n}, \varphi_{n}\right)$, где

$$
U_{n}=\bigcup_{t=0}^{h_{n}} T^{t} C_{n}, \quad h_{n}=q_{n-1} h_{n-1}+\sum_{j} s_{n-1, j}
$$

и $\varphi_{n}$ задано естественньм образом. Предъявленная конструкция задается следующими параметрами: последовательностями $h_{n}$ и $q_{n}$, а также величинами $s_{n, j}$.

Стохастическая конструкция. Будем считать, что высоты $h_{n}$ фиксированы, a $s_{n, j}$ являются независимыми случайными величинами с распределением $\xi_{n}$ (одинаковьм для всех $s_{n, j}$ при фиксированном $n$ ). При этом количество $q_{n}$ отрезков в нашей конструкции тоже случайно и определяется последовательностью $s_{n, 1}, s_{n, 2}, \ldots$ Относительно величин $\xi_{n}$ будем предполагать следующее:

a) $0 \leqslant \xi_{n} \leqslant h_{n}$

б) величина $\xi_{n}$ имеет плотность $p_{n}$, являющуюся функцией ограниченной вариации.

Обозначим $\sigma_{n}^{2}=\mathrm{D} \xi_{n}$ дисперсию величины $\xi_{n}$, и пусть $\kappa_{n}=h_{n}^{2} / \sigma_{n}^{2}$. Тогда верна

Теорема 1. Пусть поток $\left(T^{t}\right)$ является результатом предложсенной выше конструкчии, зависящей от параметров $h_{n}, \xi_{n}$, и пусть для некоторых констант $0<\chi<1$, $\gamma, \varepsilon, C_{1}, C_{2}>0$ выполнены следуюшие условия:
1) $\left\|\left(1+C_{1} \sigma_{n}^{2} x^{2}\right)^{1 / 2} \widehat{p}_{n}(x)\right\|_{\infty}=1$;
2) $\prod_{j<n} q_{j} \leqslant C_{2} q_{n}^{\gamma}$
3) $\left\|q_{n}^{-\chi}{ }_{1} h_{n} p_{n}\right\|_{\infty} \rightarrow 0$;
4) $\kappa_{n} / q_{n}^{1-\chi-\varepsilon} \rightarrow 0$;

где $\widehat{p}_{n}(x)$ - преобразование Фурье функции $p_{n}(t)$. Тогда поток $\left(T^{t}\right)$ является перемешивающим с вероятностью 1.

Приведем конкретный пример. Фиксируем распределение $\xi$ на $\mathbb{R}_{+}$с плотностью $p(t)$ и дисперсией, равной 1 , такое, что $\xi \leqslant M<\infty$. Пусть $\xi_{n}$ имеет плотность $p_{n}=p\left(t / \sigma_{n}\right) / \sigma_{n}$. И пусть также $h_{n}=2^{m^{n}}$ и $\sigma_{n}=2^{\alpha m^{n}}$. Тогда поток $\left(T^{t}\right)$ будет перемешивающим почти наверное, если $\alpha>3 /(2+m)$. 
2. Перемешивающие системы положительного локального ранга. В этом пункте мы опишем стохастическую конструкцию потока заданного локального ранга $\beta \in(0,1)$, которая в основном опирается на конструкцию потока ранга 1 , описанную в предыдущем пункте.

Фиксируем $\beta \in(0,1)$ и рассмотрим последовательность наборов башен $\left(U_{n}^{(k)}, \varphi_{n}^{(k)}\right)$ высоты $h_{n}$, $k=0, \ldots, r_{n}$, которые мы будем отождествлять с множествами $C_{n}^{(k)} \times\left[0, h_{n}\right]$. Далее, выберем, как и раньше, последовательность случайных величин $\xi_{n}$. Нетрудно построить стационарньй случайный процесс

$$
\Psi_{n}: \mathbb{R} \rightarrow\left(\left[0, h_{n}\right] \times\left\{0, \ldots, r_{n}\right\}\right) \sqcup\{\mathfrak{s}\}
$$

такой, что

a) если $\Psi_{n}(t)=(0, k)$, то $\Psi_{n}(t+u)=(t, k)$ при $0 \leqslant u \leqslant h_{n}$;

b) если $\Psi_{n}(t)=h_{n}$, то за точкой $t$ следует интервал длины $s$, на котором $\Psi_{n}(t) \equiv \mathfrak{s}$ и в правом конце которого $\Psi_{n}$ обращается в 0 , причем длины всех таких интервалов независимы и распределены как $\xi_{n}$;

c) вторая координата $\Psi_{n}(t)$, которая постоянна на каждом из отрезков, где $\Psi_{n}(t) \neq \mathfrak{s}$, является реализацией бернуллиевского процесса с алфавитом $\left\{0,1, \ldots, r_{n}\right\}$ и вероятностным вектором $\left\{\beta,(1-\beta) / r_{n}, \ldots,(1-\beta) / r_{n}\right\}$.

Возьмем $r_{n+1}+1$ независимых копий процесса $\Psi_{n}$, и пусть $\psi_{n}^{(k)}$ - "ограничение" соответствующей копии на $\left[0, h_{n+1}\right]$, понимаемое в следующем смысле. Если некоторый (максимальный) отрезок, на котором $\Psi_{n}(t) \neq \mathfrak{s}$, целиком содержится в $\left[0, h_{n+1}\right]$, то на нем $\psi_{n}^{(k)}(t)=\Psi_{n}(t)$; во всех остальных точках $\psi_{n}^{(k)}(t)=\mathfrak{s}$.

Отображения $\psi_{n}^{(k)}$ задают вложение башен $U_{n}^{(k)}$ в $U_{n+1}^{(k)}$. А именно, будем считать, что множество $T^{t} C_{n}^{(m)}$ совпадает с объединением множеств вида $T^{u} C_{n+1}^{(k)}$, удовлетворяющих условию $\psi_{n}^{(k)}(u)=(t, m)$. Нетрудно проверить, что отображения $\psi_{n}^{(k)}$ однозначно определяют поток, действующий таким образом, как описано выше. Заметим, что при определенных ограничениях на $r_{n}$ почти наверное $\mu\left(U_{n}^{(0)}\right) \sim \beta$ и $\mu\left(U_{n}^{(k)}\right) \sim(1-\beta) / r_{n}, k>0$.

Для формулировки следующей теоремы нам понадобится еще одно понятие. Caмоприсоединением (второго порядка) потока $\left(T^{t}\right)$ называется мера $\nu$ на прямом произведении $X \times X$, имеющая стандартные проекции на прямые сомножители и инвариантная относительно потока $\left(T^{t} \times T^{t}\right)$. Говорят, что поток $\left(T^{t}\right)$ обладает свойством минимальности самоприсоединений второго порядка, если все его эргодические самоприсоединения второго порядка исчерпьваются мерами $\mu \times \mu$ и $\left(T^{a} \times \mathrm{Id}\right) \Delta, a \in \mathbb{R}$, где $\Delta(A \times B)=\mu(A \cap B)$.

Теорема 2. Пусть в дополнение $к$ сказанному ранее в этом пункте $h_{n}$ и $\xi_{n}$ удовлетворяют ещ, условиям теоремы 1 и пусть $r_{n}=n$. Тогда построенный выше поток почти наверное является перемешивающим, причем

1) $\beta(T)=\beta$;

2) $\left(T^{t}\right)$ имеет бесконечный ранг;

3) $\left(T^{t}\right)$ обладает свойством минимальности самоприсоединений второго порядка.

Модифицируя рассмотренную конструкцию, можно построить примеры систем, обладающих свойством минимальности самоприсоединений и таких, что $\beta(T)=0$. Для этого нужно рассмотреть последовательность башен $\left(U_{n}^{(k)}, \varphi_{n}^{(k)}\right), k=1, \ldots, n$, аналогичную построенной выше, с той лишь разницей, что для построения процесса $\Psi_{n}$ мы берем вероятностный вектор $\left(1 / r_{n}, \ldots, 1 / r_{n}\right)$. Заметим, что все известные примеры автоморфизмов со свойством минимальности самоприсоединений являются системами ранга 1 (первая из таких конструкций была предложена Рудольфом в [5]). Среди потоков исключением являются некоторые орициклические потоки. 


\section{СПИСОК ЦИТИРОВАННОЙ ЛИТЕРАТУРЫ}

1. Ornstein D. S. // Proc. Sixth Berkley Sympos. Math. Statist. Probab. Univ. of California Press, 1970. P. 347-356. 2. el Abdalaoui H. Étude spectral des transformations d'Ornstein. Thèse de l'Université de Rouen. 3. Bourgain J. // Israel J. Math. 1993. V. 84. P. 53-63. 4. Ferenczi S. // Colloquium Mathematicum. 1997. V. 73. №1. P. 35-65. 5. Rudolph D. J. // J. d'Analyse Math. 1979. V. 35. P. $97-122$.

Московский государственный университет им. М. В. Ломоносова 